\title{
Acute effects of right ventricular pacing on cardiac haemodynamics and transvalvular impedance
}

\author{
Milos Taborsky ${ }^{a}$, Marian Fedorco ${ }^{a}$, Tomas Skala ${ }^{a}$, Eva Kocianova ${ }^{a}$, Dalibor Pastucha ${ }^{b}$, David Richter ${ }^{a}$, Jana Petrkova ${ }^{a}$, \\ Franco Di Gregorioc, Alberto Barbettac', Jan Vaclavik ${ }^{\mathrm{a}}$
}

\begin{abstract}
Aims. To assess the acute side-effects of right ventricular (RV) stimulation applied in apex and mid-septum, in order to establish the optimal lead location in clinical practice.

Methods. During pacemaker implantation, the ventricular lead was temporarily fixed in the apex and then moved to mid-septum. In both positions, surface and endocardial electrograms and transvalvular impedance (32 cases), left ventricular (LV) pressure (23), and transthoracic echocardiography (10) were acquired with intrinsic activity and VDD pacing. Results. A larger increase in QRS duration was noticed with apical than septal pacing $\left(65 \pm 25 \mathrm{vs} .45 \pm 29 \mathrm{~ms} ; P<10^{-4}\right)$. The proportion of cases where RV stimulation affected the transvalvular impedance waveform was higher with apical lead location ( $56 \%$ vs. $20 \%$; $P<0.02$ ). VDD pacing at either site reduced the maximum $\mathrm{dP} / \mathrm{dt}$ by $6 \%$ with respect to intrinsic AV conduction (IAVC; $P<0.005)$. The maximum pressure drop taking place in $100 \mathrm{~ms}$ was reduced by 6 and $8 \%$, respectively, with apical and septal pacing $(P<0.01$ vs. IAVC). Apical VDD decreased mitral annulus velocity in early diastole ( $\left.E^{\prime}\right)$ from $7.5 \pm 1.4$ to $5.9 \pm 0.9 \mathrm{~cm} / \mathrm{s}(P<0.02)$ and prolonged the E-wave deceleration time (DT) from $156 \pm 33$ to $199 \pm 54 \mathrm{~ms}(P<0.02)$, while septal pacing induced non-significant modifications in $\mathrm{E}^{\prime}$ and $\mathrm{DT}$.
\end{abstract}

Conclusion. Ventricular stimulation acutely impairs LV systolic and diastolic performance, independent of the pacing site. Septal lead location preserves RV contraction mechanics and reduces the electrical interventricular delay.

Key words: apical pacing, septal pacing, QRS duration, LV pressure, transvalvular impedance, echocardiography

Received: September 7, 2012; Accepted with revision: January 29, 2013; Available online: February 18, 2013

http://dx.doi.org/10.5507/bp.2013.009

${ }^{a}$ Department of Internal Medicine I - Cardiology, University Hospital Olomouc, Czech Republic

${ }^{b}$ Department of Exercise Medicine and Cardiovascular Rehabilitation, University Hospital Olomouc

'Clinical Research Unit, Medico Spa, Rubano (PD), Italy

Corresponding author: Jan Vaclavik, e-mail:vaclavik.j@centrum.cz

\section{INTRODUCTION}

Electrical stimulation of the ventricle is mandatory to restore properly timed activity in patients affected by atrioventricular block (AVB). However, with the energy normally used for pacing purposes, the electric pulse directly excites just a small portion of the ventricular myocardium, restricted to about $1 \mathrm{~mm}$ in case of point stimulation by a very small electrode ${ }^{1}$. Starting from the edge of this area, the activation front spreads through the myocardial cell network until Purkinje fibres are depolarized and can eventually contribute to the latest part of the conduction process. Focal pacing thus entails heterogeneous activation delays in different regions and ventricular electromechanical dyssynchrony.

The haemodynamic implications of ventricular desynchronization are a matter of investigation. Several studies have reported alterations in systolic and diastolic function associated with chronic apical pacing ${ }^{2-4}$, which could explain the increased incidence of atrial fibrillation and heart failure in long-term paced patients ${ }^{5-7}$. Milder effects on ventricular synchronization and pump function have been associated with right ventricular (RV) pacing applied in alternative sites, like the mid-septum or the outflow tract $^{2,8-10}$. These claims might have a significant impact in the clinical setting ${ }^{11}$, as pacing lead positioning in the RV apex is still normal practice in many implantation centres.

Evidence in favour of alternative site pacing is mostly based on the electrocardiographic and echocardiographic evaluation of RV stimulation side-effects, while a significant influence of the pacing site on functional capacity, quality of life and survival has not been demonstrated by randomized controlled trials ${ }^{12}$. Previous investigations relying on invasive pressure measurements reported a protective effect of high septal pacing ${ }^{13}$, or no significant worsening induced by RV stimulation either in the apex or outflow tract ${ }^{14}$. In order to test whether a septal lead location should be proposed as first option in standard pacemaker implants, the present study coupled the echocardiographic assessment of the main indices of haemodynamic function with the measurement of left ventricular (LV) pressure by cardiac catheterization in a cohort of bradyarrhythmic patients with normal ejection fraction (EF $>50 \%$ with intrinsic conduction), temporarily paced in RV apex and mid-septum. In addition, the transvalvular impedance (TVI) waveform was recorded. TVI, the electrical impedance derived between the right atrium and ventricle, changes during the cardiac cycle in a fashion strongly suggesting a correlation with RV ejection and filling ${ }^{15-17}$. TVI waveform analysis could therefore provide 
insight into pacing-induced modifications in RV mechanical activity, which is generally difficult to assess with conventional echocardiographic techniques.

\section{METHODS}

The study was approved by the local ethics committee and informed consent was obtained from the enrolled patients. The tests were carried out during the implantation of dual-chamber pacemakers for standard indications, using active fixation endocardial pacing leads Tendril $\AA$ 1882T and 1888T (St. Jude Medical, Inc., USA). The atrial lead was settled in the right appendage; the RV lead was first fixed in the apex and then definitively moved to the mid-septum. The electrode position was graphically documented and verified by X-Ray $\left(\mathrm{AP}+30^{\circ} \mathrm{LAO} / \mathrm{RAO}\right)$ and echocardiography. At each ventricular site, recordings were performed during intrinsic atrio-ventricular conduction (IAVC), AAI pacing at $90 \mathrm{bpm}$, and atrium-driven ventricular pacing. In the latter case, the AV delay was set at $80 \mathrm{~ms}$ to ensure fully evoked ventricular activation. The effects of the activation pattern switch on LV pressure, cardiac electric signals and TVI were assessed after 3-min stimulation. At the same time, the echocardiographic evaluation was started and carried out in about 15 min per each step of the test procedure (IAVC, AAI, apical VDD and septal VDD).

\section{TVI, electrograms, and LV pressure measurements}

TVI recording was performed in 32 patients with IAVC. The impedance was derived between the ring atrial electrode and either the tip or ring ventricular electrode, choosing the configuration which provided the most physiological signal at a given lead location, i.e., an impedance waveform increasing throughout the ejection phase to reach a single maximum peak at the end of the QT interval, and then decreasing back to the baseline with ventricular filling. Pacing-induced TVI modifications were considered relevant if the peak-peak TVI amplitude was reduced by more than $25 \%$ with respect to IAVC in each patient, or if the paced waveform featured more than one positive peak in the QT interval.

In 23 patients with no contraindication to invasive haemodynamic assessment, a Mikro-Tip pressure catheter (Millar, USA) was inserted through the femoral artery and positioned in the LV to allow continuous pressure measurement. The pressure amplifier output, surface ECG, atrial and ventricular electrograms, and TVI tracing were simultaneously recorded at $1-\mathrm{KHz}$ sampling rate and stored in memory by a dedicated research device (Medico Spa, Italy) for offline data processing. The following parameters were derived from the LV pressure tracing: minimum diastolic and maximum systolic pressure $\left(\mathrm{LVP}_{\min } ; \mathrm{LVP}_{\max }\right)$, maximum rate of rise in isometric systole $\left(\mathrm{dP} / \mathrm{dt}_{\max }\right)$ and rate of fall in isometric diastole $\left(\mathrm{dP} / \mathrm{dt}_{\text {min }}\right)$, maximum pressure drop taking place in $100 \mathrm{~ms}$ $\left(\Delta \mathrm{P}_{100}\right)$, also expressed as a fraction of the total pressure excursion $\left(\Delta \mathrm{P}_{100} / \mathrm{PP}\right)$.

\section{Echocardiography}

Extensive haemodynamic assessment was performed in each cardiac activation modality by transthoracic echocardiography (GE Ultrasound Vivid 7, GE Healthcare, UK). Although non-invasive, the echocardiographic approach markedly prolonged the implantation procedure and therefore could be applied in just 10 patients, selected on the basis of their compliance and the availability of a suitable acoustic window. Resting examination was achieved in apical 4-chamber $(\mathrm{A} 4 \mathrm{CH})$ and 2-chamber $(\mathrm{A} 2 \mathrm{CH})$ views with 1-lead ECG, during a calm end-expirium. All stored sequences were evaluated offline by two skilled physicians not aware of the patients' clinical status or results of other examinations. The average of 3 consecutive cardiac cycles was considered in the statistical analysis. The LV ejection fraction (EF) was calculated using Simpson's rule from $\mathrm{A} 4 \mathrm{CH}$ and $\mathrm{A} 2 \mathrm{CH}$ views. The transmitral flow was assessed by pulsed Doppler and the velocity of mitral annulus by TDI.

\section{Statistical analysis}

All parametric data are reported as means \pm standard deviation. The statistical significance of differences associated with changes in the ventricular activation modality was evaluated by the paired Student's t-test, applying the Bonferroni correction in case of multiple comparisons. The proportions of patients showing relevant TVI modifications induced by apical or septal stimulation were compared by McNemar's test for related groups.

\section{RESULTS}

\section{Electrical synchronization}

In each patient, the ventricular activation modality was switched from IAVC to VDD stimulation with the ventricular lead sequentially positioned in both RV apex and mid-septum. The apical stimulation produced an individual increase in QRS duration of $65 \pm 25 \mathrm{~ms}$, while septal pacing resulted in a smaller QRS widening (45 \pm $\left.29 \mathrm{~ms} ; P<10^{-4}\right)$. In addition, the paced QRS axis was more homogeneous and physiologically oriented with septal than apical lead location (range from 0 to $25^{\circ}$ and from -56 to $10^{\circ}$, respectively).

The relationship between the duration of the QRS complex evoked by VDD stimulation at the two ventricular sites is shown in Fig. 1. With respect to apical pacing, septal stimulation never increased the QRS duration by more than $5 \%$. The difference was limited to the range of $\pm 5 \%$ in $21 \%$ of the cases, while a substantial reduction was noticed in the rest of the group. In $58 \%$ of cases, septal stimulation decreased the paced QRS width by more than 5 to $20 \%$. In the remaining $21 \%$ of patients, the QRS was shortened to less than $80 \%$ of that produced by VDD pacing applied in the apex.

\section{Left-ventricular pressure}

When the cardiac rate was raised from the sinus rhythm $(60 \pm 14 \mathrm{bpm})$ to $90 \mathrm{bpm}$ by AAI pacing, the 
$\mathrm{dP} / \mathrm{dt}_{\max }$ increased by $15.5 \pm 11.5 \%(P<0.001)$, indicating that the inochronic autoregulation could substantially affect the haemodynamic performance in the tested patient group. Taking this in due consideration, the effects of VDD stimulation on LV pressure were evaluated only in the subgroup of patients featuring 1:1 IAVC, where the transition from sinus rhythm to VDD did not modify the cardiac rate $(n=14)$. Either apical or septal pacing produced no effect on $\mathrm{LVP}_{\max }$, while $\mathrm{LVP}_{\min }$ showed a small, yet highly significant increase (Tab. 1). Indeed, LVP $_{\text {min }}$ was enhanced in 13 out of 14 patients at both pacing sites. The individual increase with respect to IAVC was statistically significant in 9 and 7 cases with apical and septal stimulation, respectively. Switching the ventricular activation mode from IAVC to VDD also resulted in a significant $6 \%$ reduction in $\mathrm{dP} / \mathrm{dt}_{\max }$, irrespective of the pacing site (Table 1). At the individual level, a significant decrease was noticed in 11 patients out of 14 and the proportion was the same with apical and septal stimulation.

The instantaneous rate of pressure fall $\left(\mathrm{dP} / \mathrm{dt}_{\min }\right) \mathrm{did}$ provide a univocal expression of the relaxation speed, as RV stimulation often resulted in uneven pressure waveforms, featuring alternating deceleration and acceleration in the isometric diastole instead of the monotonic decrease normally seen with IAVC (Fig. 2). In contrast, the maximum pressure drop taking place in $100 \mathrm{~ms}$ $\left(\Delta \mathrm{P}_{100}\right)$ was not a reliable index of relaxation efficiency. Either apical or septal pacing significantly decreased $\Delta \mathrm{P}_{100}$ (Table 1), as well as the fraction of total pressure excursion accomplished in $100 \mathrm{~ms}\left(\Delta \mathrm{P}_{100} / \mathrm{PP}\right)$. In both stimulation modalities, a significant reduction in $\Delta \mathrm{P}_{100}$ was induced in 10 patients out of 12 .

In summary, the transition from IAVC to VDD pacing affected $\mathrm{LVP}_{\text {min }}$ and the rate of pressure change in isometric systole and diastole. No significant difference was found between apical and septal VDD.

\section{Echocardiography}

The main echocardiographic indices of systolic and diastolic function were sequentially assessed in IAVC and VDD pacing. Atrium-driven ventricular stimulation at either the RV apex or mid-septum produced a reduction in ejection fraction (EF), mitral flow peak velocity in early diastole (E), and mitral annulus systolic velocity (S), which was not statistically significant (Table 2). Mitral annulus velocity in early diastole (E') was significantly decreased by apical pacing, while septal stimulation produced a non-significant reduction. The E/E' ratio was increased by pacing at either site, but in both cases statistical significance was lacking. A significant prolongation of E-wave deceleration time (DT) was observed with apical stimulation, while septal pacing entailed a non-significant increase in DT. This aside, none of the assessed echocardiographic parameters showed a statistically significant difference in the direct comparison of apical and septal pacing effects.

\section{Trans-valvular impedance}

TVI recording was performed in 32 patients with the ventricular lead sequentially positioned in the apex and mid-septum. In the presence of IAVC, a physiological TVI waveform featuring only one positive peak was detected at both sites in 25 cases, in the apex and not septum in 4, in the septum and not apex in 1, and in neither the apex nor the septum in 2. The frequency of success was not significantly different at the two sites $(P=0.37$, McNemar's test for paired proportion). When the ventricular lead was located in the apex, TVI was derived with the ring electrode in $64 \%$ of the cases, and with the tip in the remaining $36 \%$. At the mid-septum, the ring ventricular electrode was selected for TVI recording in $58 \%$ of the cases, and the tip in the remaining $42 \%$. The amplitude of TVI excursion from diastole to end-systole as a function of recording site and configuration is reported in Tab. 3. With either tip or ring ventricular electrodes, the signal amplitude was not significantly different in the RV apex and septum.

Table 1. Acute effects of VDD pacing on LV pressure.

\begin{tabular}{|c|c|c|c|c|c|c|}
\hline & \multicolumn{3}{|c|}{ Apex } & \multicolumn{3}{|c|}{ Septum } \\
\hline & $\begin{array}{l}\text { Intrinsic } \\
\text { conduction }\end{array}$ & VDD & $P(\mathrm{t})$ & $\begin{array}{l}\text { Intrinsic } \\
\text { conduction }\end{array}$ & VDD & $P(\mathrm{t})$ \\
\hline $\begin{array}{l}\mathrm{LVP}_{\max } \\
(\mathrm{mmHg})\end{array}$ & $145 \pm 25$ & $143 \pm 27$ & n.s. & $146 \pm 30$ & $145 \pm 27$ & n.s. \\
\hline $\begin{array}{l}\mathrm{LVP}_{\min } \\
(\mathrm{mmHg})\end{array}$ & $2.9 \pm 2.1$ & $4.5 \pm 2.4$ & $<0.001$ & $2.9 \pm 2.7$ & $4.3 \pm 3.4$ & $<0.002$ \\
\hline $\begin{array}{c}\mathrm{dP} / \mathrm{dt}_{\text {max }} \\
(\mathrm{mmHg} / \mathrm{s})\end{array}$ & $1406 \pm 251$ & $1319 \pm 250$ & $<0.002$ & $1393 \pm 263$ & $1303 \pm 257$ & $<0.005$ \\
\hline $\begin{array}{c}\Delta \mathrm{P}_{100} \\
(\mathrm{mmHg})\end{array}$ & $-104 \pm 23$ & $-98 \pm 20$ & $<0.01$ & $-105 \pm 21$ & $-97 \pm 19$ & $<0.001$ \\
\hline$\Delta \mathrm{P}_{100} / \mathrm{PP}$ & $0.748 \pm 0.063$ & $0.722 \pm 0.061$ & $<0.05$ & $0.741 \pm 0.061$ & $0.699 \pm 0.050$ & $<0.02$ \\
\hline
\end{tabular}

Right ventricular stimulation was sequentially applied in the apex and mid-septum. All parameters were assessed twice with intrinsic conduction,

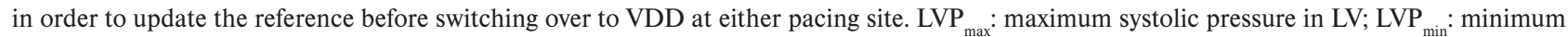
diastolic pressure in $\mathrm{LV} ; \mathrm{dP} / \mathrm{dt}_{\max }$ : maximum rate of rise of $\mathrm{LV}$ pressure; $\Delta \mathrm{P}_{100}$ : maximum LV pressure drop in $100 \mathrm{~ms} ; \mathrm{PP}: \mathrm{LV}$ pulse pressure. $P(\mathrm{t})$ values are derived by the paired Student's t-test, comparing VDD and intrinsic conduction data in each patient; n.s.: not significant $(P \geq 0.05)$. 
Table 2. Acute effects of VDD pacing on LV haemodynamic parameters.

\begin{tabular}{|c|c|c|c|c|c|}
\hline & $\begin{array}{l}\text { Intrinsic } \\
\text { conduction }\end{array}$ & $\begin{array}{l}\text { VDD } \\
\text { apex }\end{array}$ & $P(\mathrm{t})$ & $\begin{array}{c}\text { VDD } \\
\text { septum }\end{array}$ & $P(\mathrm{t})$ \\
\hline EF (\%) & $59 \pm 5$ & $56 \pm 6$ & n.s. & $55 \pm 6$ & n.s. \\
\hline $\mathrm{S}(\mathrm{cm} / \mathrm{s})$ & $8.1 \pm 1.3$ & $8.0 \pm 1.2$ & n.s. & $7.8 \pm 1.8$ & n.s. \\
\hline$E^{\prime}(\mathrm{cm} / \mathrm{s})$ & $7.5 \pm 1.4$ & $5.9 \pm 0.9$ & $<0.02$ & $6.1 \pm 1.5$ & n.s. \\
\hline $\mathrm{E}(\mathrm{cm} / \mathrm{s})$ & $75.3 \pm 24.0$ & $67.4 \pm 18.4$ & n.s. & $65.1 \pm 20.1$ & n.s. \\
\hline $\mathrm{E} / \mathrm{E}^{\prime}$ & $10.4 \pm 5.4$ & $12.1 \pm 5.0$ & n.s. & $12.1 \pm 8.1$ & n.s. \\
\hline DT (ms) & $156 \pm 33$ & $199 \pm 54$ & $<0.02$ & $188 \pm 39$ & n.s. \\
\hline
\end{tabular}

Both apical and septal ventricular stimulation is compared with intrinsic AV conduction by the paired Student's t-test and Bonferroni correction for twice repeated trials. EF: ejection fraction; S: mitral annulus systolic velocity; E': mitral annulus velocity in early diastole; E: peak mitral flow velocity in early diastole; DT: E-wave deceleration time.

Table 3. TVI peak-peak amplitude with intrinsic AV conduction.

\begin{tabular}{lll}
\hline & \multicolumn{2}{c}{ TVI amplitude (Ohm) } \\
\hline TVI config. & APEX & SEPTUM \\
V ring & $50 \pm 23$ & $47 \pm 17$ \\
V tip & $44 \pm 22$ & $35 \pm 20$ \\
\hline
\end{tabular}

The signal was recorded in the apical and septal positions with either ring or tip ventricular electrodes.

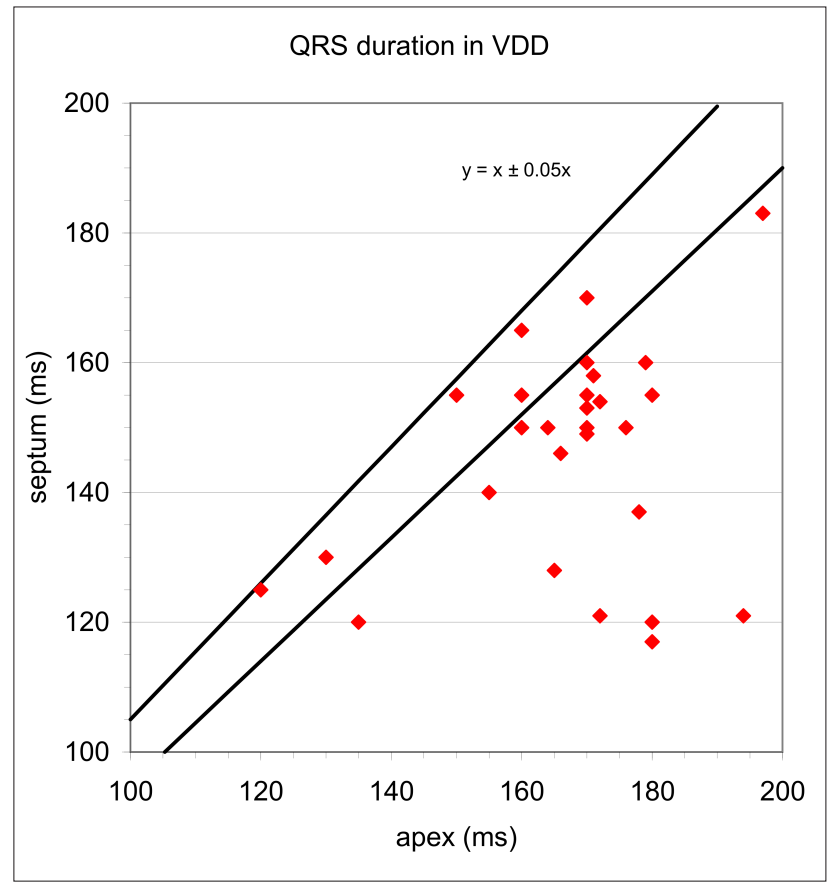

Fig. 1. Relationship between QRS duration with apical and septal VDD stimulation in each patient. The difference is higher than $5 \%$ outside the area between the two lines.
The effect of VDD pacing on the TVI waveform was evaluated in patients showing a physiological signal with IAVC at both apical and septal lead locations $(n=25)$. Relevant TVI modifications were induced by both apical and septal stimulation in 4 cases, by septal but not apical stimulation in 1 case, by apical but not septal stimulation in 10 cases, and by neither apical nor septal stimulation in 10 cases. Objective definitions of "physiological TVI waveform" and "relevant TVI modifications" are provided in the Methods section. The proportion of patients featuring TVI deterioration was significantly higher with apical than septal pacing $(56 \%$ vs. $20 \%, P<0.02)$. A representative example of TVI response to ventricular stimulation is shown in Fig. 3.

\section{DISCUSSION}

It is widely recognized that long-lasting pacing treatment with a high incidence of RV apical stimulation can reduce the haemodynamic performance and increase the risk of atrial fibrillation and heart failure ${ }^{2-7}$. Although the mechanisms involved in pacing-related damage are not fully understood, it is conceivable that functional and structural deterioration could result from the increased myocardial stress induced by ventricular desynchroniza$\operatorname{tion}^{10,18}$. The acute haemodynamic consequences of ventricular stimulation could thus predict the possibility of permanent haemodynamic impairment in chronic conditions and help choose the site where pacing is best tolerated in each patient.

\section{Left ventricular haemodynamics}

With the aim of maximizing sensitivity and specificity to pacing-induced LV pressure changes, the present study compared relevant pressure parameters right before and after a switch in ventricular activation modality. The time lag between two paired measurements never exceeded 4 min, strongly reducing any bias due to slow changes in basal haemodynamics or in the response of the recording set-up. The transition from IAVC to sequential RV pacing at a constant rate entailed a significant decrease in $\mathrm{dP} /$ $\mathrm{dt}_{\max }$ and $\Delta \mathrm{P}_{100}$, a pressure derived index of the isometric relaxation speed. These results are consistent with a pre- 


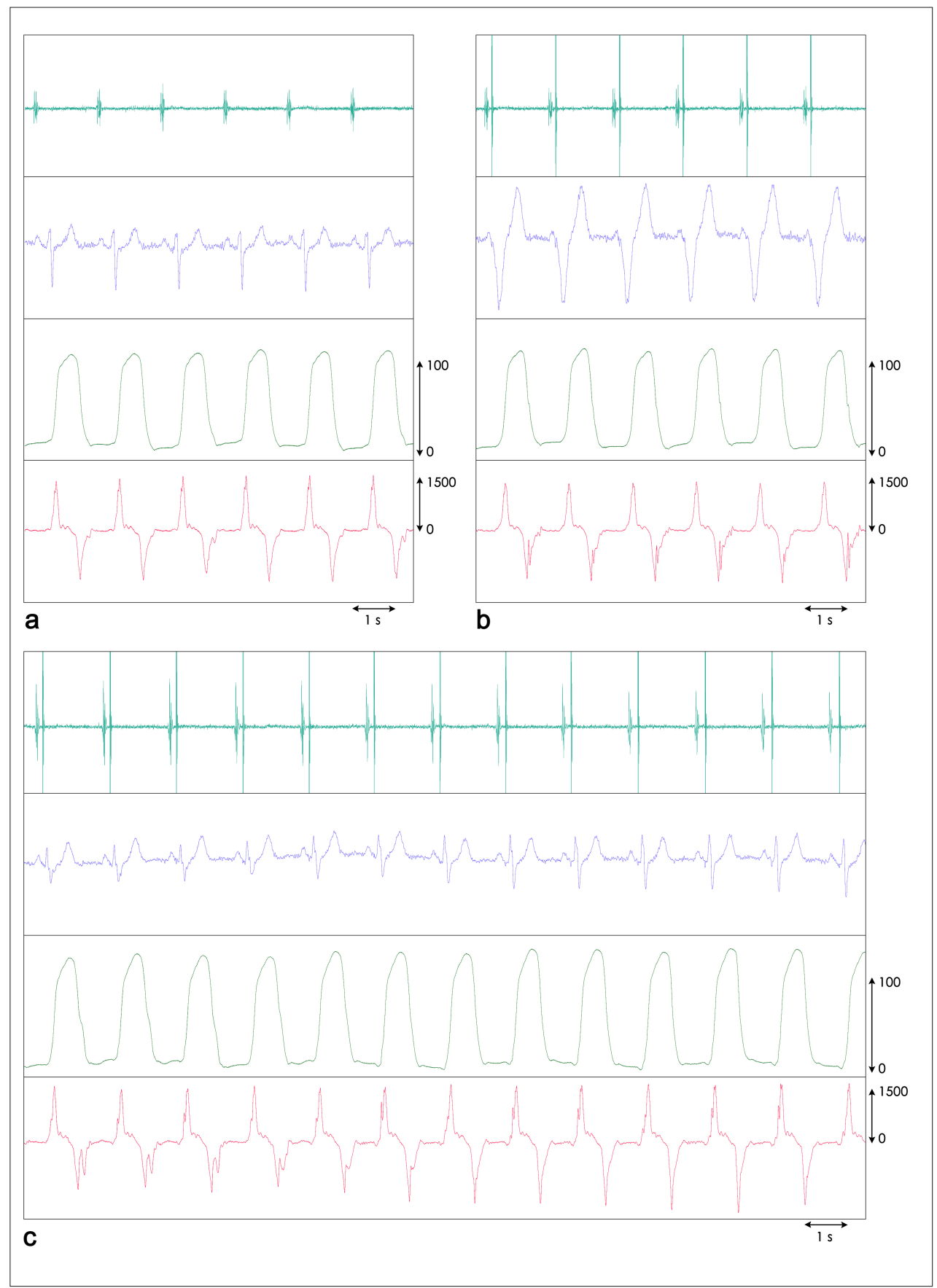

Fig. 2. From top to bottom tracings: AEGM, ECG (II), LVP (the arrows mark the range from 0 to $100 \mathrm{mmHg}$ ), $\mathrm{LV} \mathrm{dP} / \mathrm{dt}$ (the arrows mark the range from 0 to $1500 \mathrm{mmHg} / \mathrm{s}$ ) in sinus rhythm (a) and VDD pacing with apical (b) and septal stimulation (c) in one patient. The horizontal arrows indicate a 1-s time interval. The AV delay was constant at $80 \mathrm{~ms}$ in panel b, while it was gradually increased starting from $80 \mathrm{~ms}$ in c. Axis and duration of the QRS complex were heavily affected by apical stimulation, while mild QRS changes were produced by septal pacing. In spite of different electrical effects, pacing at either site resulted in decreased $\mathrm{dP} / \mathrm{dt}_{\text {max }}$ and uneven pressure fall, as shown by the alterations in the negative portion of the $\mathrm{dP} / \mathrm{dt}$ waveform. Such modifications were readily reversed when the evoked ventricular activation was replaced by fusion beats (c).

vious work showing an increased RV and LV myocardial performance index in patients chronically paced in the $\mathrm{RV}$ ape ${ }^{19}$ and indicate a reduction in both systolic and diastolic efficiency associated with RV pacing. In our experience, the diastolic fall in LV pressure was characterized by a first time-derivative with monotonic course in IAVC. With RV pacing, in contrast, the $\mathrm{dP} / \mathrm{dt}$ tracing often showed alternating deceleration and acceleration during the isometric diastole, as depicted in Fig. 2. Such anomalous behaviour was readily reversed by stopping ventricular stimulation and therefore cannot be ascribed to a bad position or poor stability of the pressure catheter. Nevertheless, the possibility must be considered of either a true uneven pressure decrease, with pauses along its 


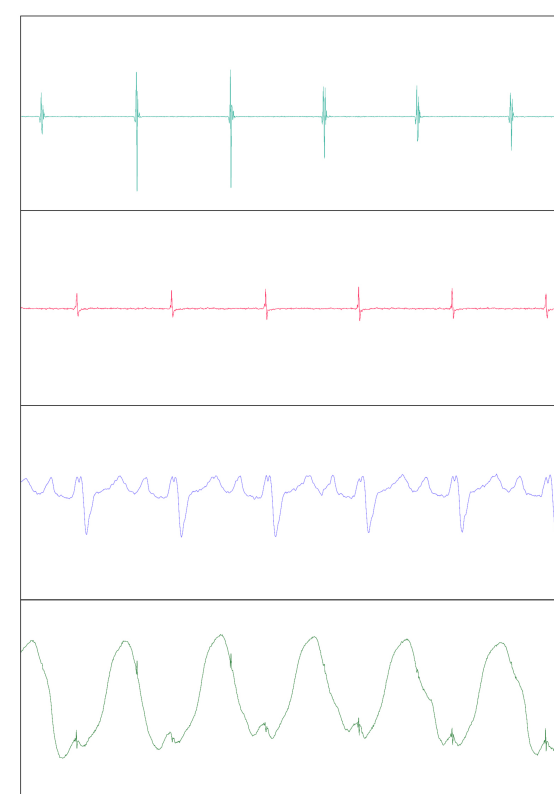

a

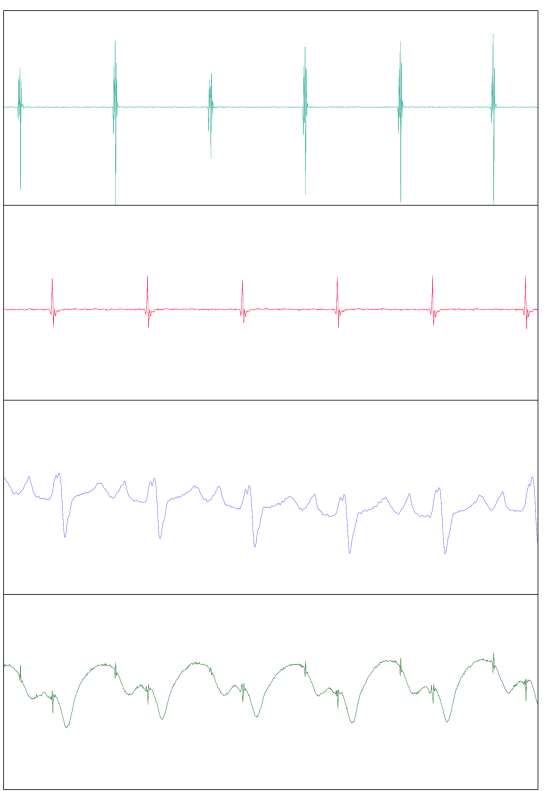

C

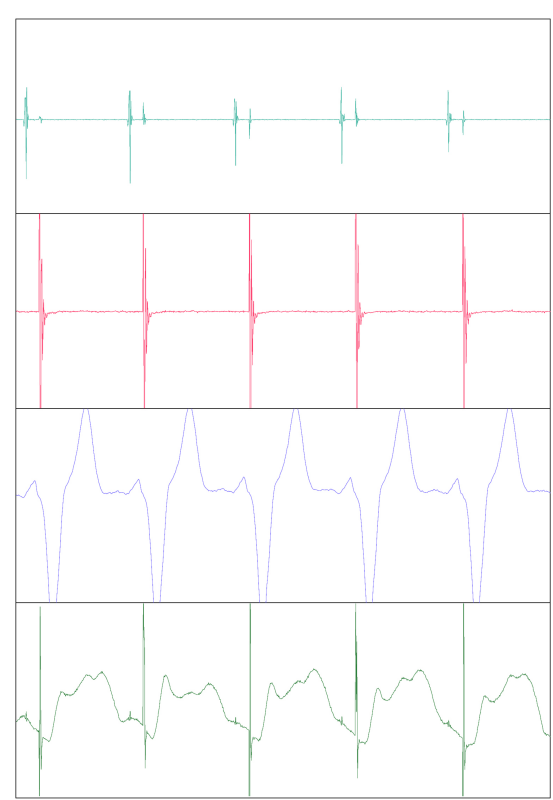

b

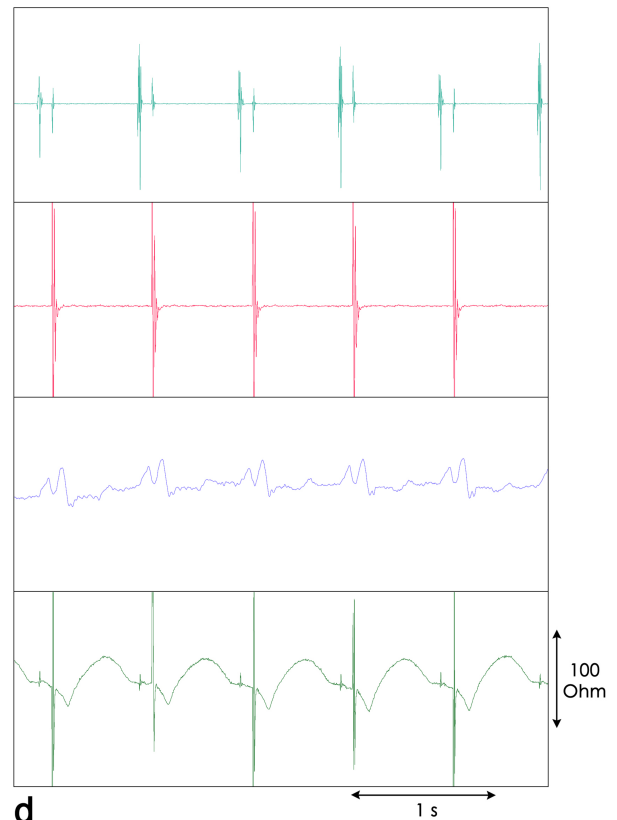

Fig. 3. From top to bottom tracings: AEGM, VEGM, ECG (II), TVI (the vertical arrows indicate a $100 \mathrm{Ohm}$ excursion and the same scale applies to all panels), recorded in one patient in sinus rhythm with intrinsic AV conduction (a, c) and VDD pacing (b, d). The horizontal arrows mark a 1-s time interval. The ventricular lead was positioned in the RV apex and mid-septum in the upper $(a, b)$ and lower panels (c, d), respectively. In both conditions, TVI was derived between the atrial and ventricular ring electrodes. With intrinsic AV conduction, the signal featured a progressive rise and only one positive peak in the QT interval. Apical stimulation (b) induced deep changes in TVI waveform, with the appearance of three peaks in the QT. In contrast, septal pacing (d) did not entail relevant TVI modifications.

course due to heterogeneous relaxation, or of artifacts generated by the contact between the pressure sensor and some LV structures only in the presence of ventricular stimulation. Artifacts of this kind would anyhow be the expression of abnormal LV movements in isometric conditions, which could only be explained by diastolic desynchronization.
The role of diastolic desynchronization in the development of pacing-induced dysfunction is controversial. On the basis of TDI cross-correlation analysis, it has been claimed that apical pacing produces systolic dyssynchrony with preserved diastolic synchrony ${ }^{20}$. However, in agreement with previous studies ${ }^{13,21}$, our experience indicates that a significant diastolic impairment acutely results from 
ventricular pacing, affecting both the isometric relaxation and the filling process. Indeed, $\mathrm{LVP}_{\text {min }}$ and E-wave DT were increased, and E' was reduced. Although the ratio $\mathrm{E} / \mathrm{E}$ ' was increased in non-significant fashion, it is conceivable that the changes in diastolic function could entail a filling pressure rise in chronic conditions, which might explain the increased incidence of atrial fibrillation associated with pacing therapy. Moreover, a reduced diastolic efficiency coupled with systolic desynchronization might enhance the cardiac stress and the risk of heart failure in the long-term.

The acute effects of RV pacing on LV haemodynamics were similar with apical or septal stimulation. No significant difference was demonstrated in the comparison between the alternative pacing sites, even though the changes in E' and DT with respect to IAVC were only significant for the apical pacing (Table 2).

\section{Right ventricular activity}

Although several studies have evaluated the acute and chronic effects of RV pacing on LV desynchronization and haemodynamic performance ${ }^{2-4,8,10}$, little is known about the RV itself ${ }^{14}$. This lack of information is partially explained by the greater attention generally paid to left heart haemodynamics, but can also result from the limited echocardiographic methods available for RV evaluation. TVI recording can be considered as a potential practical alternative, as the waveform is sensitive to the RV mechanical activity and can easily be derived by means of standard pacing leads during the implantation procedure, as well as in the follow-up of permanent implants ${ }^{15-17}$. The present study demonstrates that the TVI signal is strongly affected by RV pacing in some patients and not in others. It seems conceivable to hypothesize that marked TVI alterations might reflect corresponding modifications in RV local mechanics, whereas milder changes could be associated with a preserved contraction pattern. Interestingly, the prevalence of major TVI alterations was substantially higher with apical than septal pacing, suggesting that the latter could be less detrimental to the RV function.

\section{Interventricular versus intraventricular desynchronization}

Septal stimulation also reduced the QRS widening and improved the axis with respect to apical pacing. The difference was highly significant and evident in the vast majority of patients, in accordance with previous reports ${ }^{22,23}$. The most relevant acute benefits of septal pacing thus concerned the RV contraction properties and the overall ventricular conduction time, which greatly depends on the interventricular delay. In contrast, smaller effects of the pacing site were noticed on LV haemodynamics, which is quite sensitive to the intraventricular desynchronization. It is noteworthy that, in recent studies, pacing at the midseptum ${ }^{10}$ or outflow tract ${ }^{24}$ reduced interventricular and longitudinal LV desynchronization, without significant improvements in septal-posterior and septal-lateral wall motion delays. Most reports showing that LV function was better preserved by non-apical RV stimulation refer to chronic pacing ${ }^{2,9,18}$. Acute studies yielding positive results ${ }^{10,13}$ were performed with lead location criteria, pacing mode and duration, or AV delay setting different from the conditions applied in the present investigation, so that a comparison of the outcomes could be misleading. Moreover, the role of the pacing site in the development of LV haemodynamic dysfunction was not confirmed in some instances ${ }^{22,24-26}$. In contrast, the relevance of alternative site pacing to the interventricular electrical synchronization, as expressed by QRS duration, is well established and further strengthened by the present results.

\section{Limitations}

It is conceivable that the protective influence of septal stimulation versus acute modifications in the RV contraction pattern and interventricular delay could also preserve the heart from structural and functional remodelling in the long term. The evaluation of this, however, was beyond the aims of the present study.

The different impact on TVI waveform of pacing in the apex or mid-septum suggests that septal stimulation helps maintain a physiological contraction pattern in RV. However, the sites of ventricular pacing and TVI recording necessarily changed together when the lead was moved from the apex to the septum. It cannot be excluded that TVI sensitivity to RV mechanical modifications might be different in different recording positions.

In the discussion it should be noted that for confirmation of the influence of pacing on the interventricular septal delay the optimal measure would be the interval from the point of pacing to the earliest endocardial activation in the left ventricle. This can only be implemented by using $3 \mathrm{D}$ mapping systems that can precisely measure the local electrical activation and interpret the relationship of each segment in the left and right ventricle.

\section{CONCLUSIONS}

Right ventricular apical pacing acutely impaired LV systolic and diastolic function, increased the QRS duration and produced marked alterations in the TVI signal, suggesting relevant changes in the RV contraction pattern. Septal stimulation significantly reduced the QRS widening and the prevalence of pacing-associated TVI modifications, showing smaller effects on LV pressure and echocardiographic parameters. Apical pacing should be avoided in clinical practice, especially in patients with a large QRS prolongation or a serious deterioration of the TVI waveform.

\section{ACKNOWLEDGEMENT}

Authorship contributions: MT, FDG, AB: literature search; MT, MD, PD, JV: manuscript writing; MT: study design; MT, MF, TS, EK, DP, DR, JP, FDG, AB: data collection; MT, FDG, AB, JV: data analysis; MT, MF, TS, EK, FDG, AB, JV: data interpretation; FDG, AB: statistical analysis, figures; MT, MF, TS, EK, DP, DR, JP, FDG, $\mathrm{AB}, \mathrm{JV}$ : final approval.

Conflict of interest statement: A.B. and F.D.G. are employees of Medico Spa. 


\section{REFERENCES}

1. Wikswo JP Jr., Lin SF, Abbas RA. Virtual electrodes in cardiac tis sue: a common mechanism for anodal and cathodal stimulation. Biophysical Journal 1995;69:2195-2210.

2. Tse HF, Yu C, Wong KK, Tsang V, Leung YL, Ho WY, Lau CP. Functiona abnormalities in patients with permanent right ventricular pacing The effect of sites of electrical stimulation. Journal of the American College of Cardiology 2002;40:1451-8.

3. Thambo JB, Bordachar $P$, Garrigue $S$, Lafitte $S$, Sanders $P$, Reuter $S$, Girardot R, Crepin D, Reant P, Roudaut R, Jaïs P, Haïssaguerre $\mathrm{M}$, Clementy J, Jimenez $M$. Detrimental ventricular remodeling in patients with congenital complete heart block and chronic right ventricular apical pacing. Circulation 2004;110:3766-72.

4. O'Keefe JH Jr., Abuissa H, Jones PG, Thompson RC, Bateman TM, McGhie Al, Ramza BM, Steinhaus DM. Effect of chronic right ventricular apical pacing on left ventricular function. American Journal of Cardiology 2005;95:771-3.

5. Sweeney MO, Hellkamp AS, Ellenbogen KA, Greenspon AJ, Freedman RA, Lee KL, Lamas GA; MOde Selection Trial Investigators. Adverse effect of ventricular pacing on heart failure and atrial fibrillation among patients with normal baseline QRS duration in a clinical trial of pacemaker therapy for sinus node dysfunction. Circulation 2003;107:2932-7.

6. Nielsen JC, Kristensen L, Andersen HR, Mortensen PT, Pedersen OL, Pedersen AK. A randomized comparison of atrial and dual-chamber pacing in 177 consecutive patients with sick sinus syndrome. Journal of the American College of Cardiology 2003;42:614-23.

7. Zhang XH, Chen $\mathrm{H}$, Siu CW, Yiu KH, Chan WS, Lee KL, Chan HW, Lee SW, Fu GS, Lau CP, Tse HF. New-onset heart failure after permanent right ventricular apical pacing in patients with acquired high-grade atrioventricular block and normal left ventricular function. Journa of Cardiovascular Electrophysiology 2008;19:136-41.

8. Schwaab B, Fröhlig G, Alexander C, Kindermann M, Hellwig N Schwerdt $\mathrm{H}$, Kirsch CM, Schieffer $\mathrm{H}$. Influence of right ventricular stimulation site on left ventricular function in atrial synchronous ventricular pacing. Journal of the American College of Cardiology 1999;33:317-23.

9. Bourke JP, Hawkins T, Keavey P, Tynan M, Jamieson S, Behulava R Furniss SS. Evolution of ventricular function during permanent pacing from either right ventricular apex or outflow tract following AVjunctional ablation for atrial fibrillation. Europace 2002;4:219-28.

10. Alhous MHA, Small GR, Hannah A, Hillis GS, Broadhurst P. Impact of temporary right ventricular pacing from different sites on echocardiographic indices of cardiac function. Europace 2011;13:1738-46.

11. Manolis AS. The deleterious consequences of right ventricular apical pacing: time to seek alternate site pacing. Pacinging and Clinical Electrophysiology 2006;29:298-315.

12. Shimony A, Eisenberg MJ, Filion KB, Amit G. Beneficial effects of right ventricular non-apical vs. apical pacing: a systematic review and meta-analysis of randomized-controlled trials. Europace 2011;14:8191.

13. Karpawich PP, Mital S. Comparative left ventricular function following atrial, septal, and apical single chamber heart pacing in the young. Pacing and Clinical Electrophysiology 1997;20[Pt. I]:1983-8.
14. Buckingham TA, Candinas $R$, Attenhofer C, Van Hoeven $H$, Hug $R$, Hess O, Jenni R, Amann FW. Systolic and diastolic function with alternate and combined site pacing in the right ventricle. Pacing and Clinical Electrophysiology 1998;21:1077-84.

15. Di Gregorio F, Morra A, Finesso M, Bongiorni MG. Transvalvular impedance (TVI) recording under electrical and pharmacological cardiac stimulation. Pacing and Clinical Electrophysiology 1996;19[Pt. II]:1689-93.

16. Gasparini G, Curnis A, Gulizia M, Occhetta E, Corrado A, Bontempi L, Mascioli G, Maura Francese G, Bortnik M, Magnani A, Di Gregorio F, Barbetta A, Raviele A. Rate-responsive pacing regulated by cardiac haemodynamics. Europace 2005;7:234-41.

17. Taborsky M, Kupec J, Vopalka R, Barbetta A, Di Gregorio F. Leftventricular mechanical activity detected by impedance recording. Europace 2010;12:534-9.

18. Occhetta E, Bortnik M, Magnani A, Francalacci G, Piccinino C, Plebani $\mathrm{L}$, Marino P. Prevention of ventricular desynchronization by permanent para-Hisian pacing after atrioventricular node ablation in chronic atrial fibrillation: a crossover, blinded, randomized study versus apical right ventricular pacing. Journal of the American College of Cardiology 2006;47:1938-45.

19. Ichiki H, Oketani N, Hamasaki S, Ishida S, Kataoka T, Ogawa M, Saihara K, Okui H, Fukudome T, Shinasato T, Kubozono T, Ninomiya Y, Matsushita T, Otsuji Y, Tei C. Effect of right ventricular apex pacing on the Tei index and brain natriuretic peptide in patients with a dual-chamber pacemaker. Pacing and Clinical Electrophysiology 2006;29:985-90.

20. Fornwalt BK, Cummings RM, Arita T, Delfino JG, Fyfe DA, Campbell RM, Strieper MJ, Oshinski JN, Frias PA. Acute pacing-induced dyssynchronous activation of the left ventricle creates systolic dyssynchrony with preserved diastolic synchrony. Journal of Cardiovascular Electrophysiology 2008;19:483-8.

21. Zile MR, Blaustein AS, Shimizu G, Gaasch WH. Right ventricular pacing reduces the rate of left ventricular relaxation and filling. Journal of the American College of Cardiology 1987;10:702-9.

22. Kypta A, Steinwender C, Kammier J, Leisch F, Hofmann R. Long-term outcomes in patients with atrioventricular block undergoing septal ventricular lead implantation compared with standard apical pacing. Europace 2008;10:574-9.

23. Cano O, Osca J, Sancho-Tello MJ, Sánchez JM, Ortiz V, Castro JE, Salvador A, Olagüe J. Comparison of effectiveness of right ventricular septal pacing versus right ventricular apical pacing. American Journal of Cardiology 2010;105:1426-32.

24. Wang F, Shi H, Sun Y, Wang J, Yan Q, Jin W, Zhang J, Meng W, Zhang F, Chen G, Sun B. Right ventricular outflow pacing induces less regional wall motion abnormalities in the left ventricle compared with apical pacing. Europace 2012;14:351-7.

25. ten Cate TJF, Scheffer MG, Sutherland GR, Verzijlbergen JF, van Hemel NM. Right ventricular outflow and apical pacing comparably worsen the echocardiographic normal left ventricle. European Journal of Echocardiography 2008;9:672-7.

26. $\mathrm{Ng} \mathrm{AC,} \mathrm{Allman} \mathrm{C,} \mathrm{Vidaic} \mathrm{J,} \mathrm{Tie} \mathrm{H,} \mathrm{Hopkins} \mathrm{AP,} \mathrm{Leung} \mathrm{DY.} \mathrm{Long-term} \mathrm{im-}$ pact of right ventricular septal versus apical pacing on left ventricular synchrony and function in patients with second- or third-degree heart block. American Journal of Cardiology 2009;103:1096-101. 